\title{
Self-consistent spectra from GRMHD simulations with radiative cooling A link to reality for $\mathrm{Sgr} \mathrm{A}^{*}$
}

\author{
Samia Drappeau ${ }^{1}$, Salomé Dibi ${ }^{1}$, Sera Markoff ${ }^{1}$ and Chris Fragile ${ }^{2}$ \\ ${ }^{1}$ Sterrenkundig Instituut 'Anton Pannekoek', Universiteit van Amsterdam, \\ Postbus 94249, 1090GE Amsterdam, the Netherlands \\ email: s.drappeau@uva.nl, s.dibi-rousselle@uva.nl, s.markoff@uva.nl \\ ${ }^{2}$ Dept. of Physics \& Astronomy, College of Charleston, \\ Charleston, USA \\ email: fragilep@cofc.edu
}

\begin{abstract}
Cosmos++ (Anninos et al 2005) is one of the first fully relativistic magneto-hydrodynamical (MHD) codes that can self-consistently account for radiative cooling, in the optically thin regime. As the code combines a total energy conservation formulation with a radiative cooling function, we have now the possibility to produce spectra energy density from these simulations and compare them to data. In this paper, we present preliminary results of spectra calculated using the same cooling functions from 2D Cosmos ++ simulations of the accretion flow around Sgr A*. The simulation parameters were designed to roughly reproduce Sgr A*'s behavior at very low $\left(10^{-8}-10^{-7} \mathrm{M}_{\odot} / \mathrm{yr}\right)$ accretion rate, but only via spectra can we test that this has been achieved.
\end{abstract}

Keywords. Sgr A*, MHD, radiation mechanisms: general, plasmas, radiative transfer, diffusion, acceleration of particles.

\section{Methodology}

Given some characteristics like temperature, mass density and magnetic pressure from the simulation, we can generate broad-band spectra with emission coming from Bremsstrahlung, Synchrotron and Compton effects. These signatures can then be compared to data (Fig. 1).

Simulation characteristics are time-averaged over the last two stable orbits before generating a spectrum. We then obtain a steady-state emission spectrum to compare to data. Five models have been simulated, given these initial conditions:

1. No cooling, no spin, 1-loop magnetic field

2. No cooling, spin $=0.99$, 1-loop magnetic field

3. Cooling, spin $=0.99$, 1-loop magnetic field

4. No cooling, no spin, 4-loop magnetic field

5. No cooling, spin $=0.99$, 4-loop magnetic field

\section{Preliminary results}

This work is still in development. Further simulations of Sgr A* are ongoing. But from our first results we can already draw some interesting conclusions:

1. Synchrotron emissions are higher than expected. This divergence between models and data is due to temperature and magnetic field simulations values, 2 orders of magnitudes higher than typical Sgr A* values. 


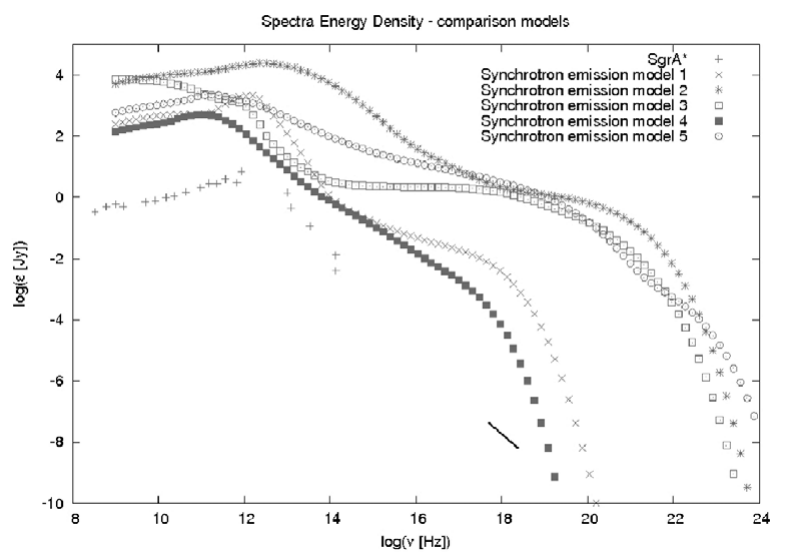

Figure 1. Sgr A* data (plus symbol and line) from Melia \& Falcke (2001). We show here synchrotron emission from no cooling, no spin, 1-loop magn. field model (cross), from no cooling, spin $=0.99,1$-loop magn. field model $($ star $)$, cooling, spin $=0.99$, 1-loop magn. field model $($ dot square), no cooling, no spin, 4-loop magn. field model (filled square) and no cooling, no spin, 4-loop magn. field model (dot circle).

2. Bremsstrahlung emission is too faint, as expected, most of the X-rays are dominated by cooler bremsstrahlung emission from the outer radii (e.g. Quataert 2002), a part of the accretion disk which is not simulated presently.

3. We did not discriminate parts of simulation known to be not realistic which results in none-physical emission, e.g. the tail in synchrotron emission might be coming from the base of the "jet", an area not well handled by the simulation.

4. Turning on cooling routines makes the emission to dramatically drop in frequency range where the accretion disk is radiating.

5. Tuning black hole spin to maximum generates too much magnetic field, hence too much emission.

\section{Conclusion}

These spectra show Dibi et al. (see proceedings article, this volume) first set of runs are a good start in modeling Sgr A* even though they are generating too much power. We have great expectations in models 1 and 4 and it will be interesting to see how their spectra behave when cooling routines are turned on. Future works also include being able to isolate emission coming from parts of the simulation we know can not be trust. And in general we want to be able to designate regions where originate each part of the emission.

\section{References}

Anninos, P., Fragile, P. C., \& Salmonson, J. D. 2005, ApJ, 635, 723

Blumenthal, G. R. \& Gould, R. J. 1970, Review of Modern Physics, 42, 237

Esin, A. A., Narayan, R., Ostriker, E., \& Yi, I. 1996, ApJ, 465, 312

Fragile, P. C. \& Meier, D. L. 2009, ApJ, 693, 771

Mahadevan, R., Narayan, R., \& Yi, I. 2009, ApJ, 465, 327

Melia, F. \& Falcke, H. 2001, ARAA, 39, 309

Quataert, E. 2002, ApJ, 575, 855

Stepney, S. \& Guilbert, P. W. 1983, MNRAS, 204, 1269 